1. MBBS, FCPS

Assistant Professor Nephrology Jinnah Postgraduate Medical Center Karachi, Pakistan.

2. MBBS, FCPS

Senior Registrar Internal Medicine

Sir Syed College of Medicine for Girls, Karachi, Pakistan.

3. MBBS

Postgraduate Trainee Nephrology Jinnah Postgraduate Medical Cente Karachi, Pakistan

4. MBBS

Postgraduate Trainee Nephrology Jinnah Postgraduate Medical Center Karachi, Pakistan.

5. MBBS

Postgraduate Trainee Medicine Jinnah Postgraduate Medical Center Karachi, Pakistan.

6. MBBS, FCPS

Professor and Head Medicine Jinnah Postgraduate Medical Center Karachi, Pakistan,

Correspondence Address:

Dr. Muhammad Ali

Room No 11B, 96 Doctor's Hostel

Jinnah Postgraduate Medical Center

Karachi, Pakistan.

maunar2002@gmail.com.

Article received on:

30/11/2019

Accepted for publication:

03/02/2020

\section{MORTALITY AMONG ORGANOPHOSPHATE POISONING PATIENTS PRESENTING WITH LOW GLASGOW COMA SCALE SCORE AT A TERTIARY CARE HOSPITAL.}

\author{
Muhammad Ali ${ }^{1}$, Vinod Kumar ${ }^{2}$, Shafique Ahmed ${ }^{3}$, Hina Iram ${ }^{4}$, Sagheer Ahmed ${ }^{5}$, \\ Rukhsana Abdul Sattar 6
}

ABSTRACT... Objectives: This study is designed to assess the mortality in OPP patients with low GCS scores. Study Design: Cross Sectional study. Setting: Jinnah Postgraduate Medical Center. Period: From 1st February 2018 to 31st August 2018. Material \& Methods: Included patients with low GCS, both genders and age of 18-60 years diagnosed with OPP. Their GCS was calculated and the outcome was determined in terms of mortality. Results: Out of 70 patients, the mean age was $35.2+16.5$ years with the majority $(62.9 \%)<30$ years. $62.9 \%$ of these were males. Most (57.1\%) of them presented after ingestion of $<15 \mathrm{~mL}$ of OP, $60 \%$ had the poisoning for $>60$ minutes, and the majority $(74.3 \%)$ had taken it orally. When the GCS was computed, $81.40 \%$ had that of $>5$, while the mean came out to be $6.64 \pm 1.43$. The mortality rate here was $17.10 \%$. Conclusion: GCS toll can be a helpful and practical tool in assessing the mortality among the patients of OPP. However, because of the limited literature on the subject further studies are recommended to improve its validity.
Key words: Glasgow Coma Scale, Mortality, Organophosphorus Compounds, Organophosphate Poisoning.
Article Citation: Ali m, Kumar V, Ahmed S, Iram H, Ahmed S, Sattar RA. Mortality among Organophosphate Poisoning Patients Presenting With Low Glasgow Coma Scale Score at a Tertiary Care Hospital. Professional Med J 2020; 27(10):2187-2192. DOI: 10.29309/TPMJ/2020.27.10.4383

\section{INTRODUCTION}

It is a well-known fact that during the last few decades, agricultural organophosphate compounds (pesticides) have become a common household item not only in the developed world but also in rural areas of the developing world. ${ }^{1}$ Due to the easy availability, these are also being commonly used for intentional self-poisoning as well as a cause of accidental poisoning. ${ }^{2}$

According to the World Health Organization, one million serious accidental and two million suicidal poisonings with organophosphates (OP) occur worldwide every year, and of these, approximately 200,000 die, mostly in developing countries. ${ }^{3,4}$ Thus organophosphate poisoning (OPP) is a significant public health problem. Since Pakistan is mainly an agricultural country, organophosphorus compounds are widely and easily available even at ordinary shops in Pakistan. ${ }^{5}$ Therefore the risk of accidental/ suicidal OPP is too high. According to a study by Shaikh MA et al., among all poisoning cases, OP ingestion was the most common mode of poisoning noted in 55\% cases with the mortality rate of $19.5 \% .{ }^{5}$ Further in Pakistan, OP poisoning was observed in $47 \%$ and $51 \%$ of accidental and suicidal cases respectively according to Balouch G et al. ${ }^{6}$

Clinical features of OPP includemiosis, hypersalivation, wheezing and drowsiness in $100 \%$ patients, chest crepitations in $61 \%$, hypotension in $72 \%$, lacrimation in $91 \%$, abdominal pain in $69 \%$ and cyanosis in $34 \%$. A mechanical ventilator is required in a quarter of patients due to respiratory failure. ${ }^{5}$ Since respiratory failure is due to central nervous system depression and is the most common cause of death in OP poisoning, early identification through neurologic assessment and effective management will help to reduce the overall mortality. ${ }^{6}$

After the first time reported use in 1974 by Graham 
Teasdale, the Glasgow coma scale (GCS) became a very efficient clinical score in assessing the consciousness of emergency patients. ${ }^{7}$ Since the last 40 years, GCS is used in several emergency departments as an indicator of the status of the central nervous system regardless of their primary etiology. ${ }^{8}$ The GCS remains the most reliable method for the assessment and prognosis of patients with non-traumatic coma ${ }^{9}$, such that it is also used in assessing the patients of OPP because these biochemical substances also affect the brain and alter the consciousness level. The GCS has been used for the outcome and recovery evaluation of patients admitted to ICU following OPP and for predicting acute and delayed outcomes. ${ }^{10}$

Despite good treatment options of OPP, the mortality is not predicted. Therefore, assessing the risk of mortality is important to allow proper resource allocation and therapeutic aggressiveness. The laboratory methods are not readily available and their usefulness remains controversial. This raises the need for a simple clinically-based prognostic system that will be more useful in resource-limited settings like ours. A Solution to this problem is the use of the Glasgow Coma Scale. The predictability of GCS among OPP patients has not been studied in the local context. Therefore this study is designed to assess the magnitude of mortality in OP poisoning patients with low GCS score $(\leq 8)$ a noninvasive modality. If mortality is found to be higher, further studies could be undertaken to assess its diagnostic validity and prognosis and at the same time counsel attendants regarding their patients.

\section{MATERIAL \& METHODS}

This is a descriptive cross-sectional study in which data were collected in the National poisoning treatment Center Medical Unit-I, ward 5, Jinnah postgraduate medical centre (JPMC), Karachi, Pakistan from $1^{\text {st }}$ February to $31^{\text {st }}$ August 2018. Using the World health organization's sample size calculator taking the estimated prevalence of the death rate among patients of OPP with low GCS score as $17.39 \%^{11}$, the sample size came out to be 68 . Patients of either gender and age of 18-60 years diagnosed with OPP by senior on-call internal medicine resident and confirmed later by the medical consultant were included. Legal formalities were done with a Medico-legal officer at the emergency department. Valid written consent was given by either patient or next of kin and all patients with GCS $<8$ were registered. An alcoholic or other poisoning cases like that of Opioids, benzodiazepine, nicotine poisoning, and Botulinum poisoning, patients under drug abuse of addiction, pediatric or geriatric patients, all were excluded. Patients having chronic diseases like diabetes mellitus, cardiovascular, respiratory, and/or hepatic failure were not considered for data collection. Even all those with metabolic acidosis due to acute/chronic renal failure or chronic obstructive pulmonary diseases were not incorporated. All the patients being partially treated, referred, or brought dead were also excluded. After approval from the ethical review committee of JPMC and the College of Physicians and Surgeons, Pakistan, informed consent was taken from the patient or immediate relative. The data was gathered on a questionnaire regarding age, gender and other variables like quantity, duration, route of administration of OP compound. The patients were followed until the next 24 hours in the ward. The final outcome (outcome variable) in terms of death or survival was recorded. The principal investigator collected all relevant data. Data entry and analysis were performed using IBM SPSS Statistics for Windows, Version 19.0 (IBM Corp., Armonk, NY). Mean ( \pm SD) was expressed for continuous variables while frequency and percentages were calculated for categorical variables. Stratification by age, gender, duration, route, and quantity of OP compound was done to see the effect of these on the outcome, A chisquare test was applied and a P-value $\leq 0.05$ taken as significant.

\section{RESULTS}

In our data of 70 patients with OPP, the mean age came out to be $35.2+16.5$ years with the majority $(n=44,62.9 \%)$ lying under the age of 31 years, with the rest greater than 30 years. $62.9 \%$ (44) of these were males and the rest were females.

When the details of poisoning were evaluated, 
most $(57.1 \%)$ of them presented after ingestion of $<15 \mathrm{~mL}$ of OP, $60 \%$ had the poisoning for $>60$ minutes, and the majority $(74.3 \%)$ had taken it via the oral route (Table-I).

\begin{tabular}{|c|c|c|}
\hline & Frequency & Percentage \\
\hline \multicolumn{3}{|c|}{$\begin{array}{l}\text { Quantity of organophosphate poisoning } \\
\qquad(\text { Mean }=13.71 \pm 9.10 \mathrm{ml})\end{array}$} \\
\hline$<15 \mathrm{ml}$ & 40 & $57.1 \%$ \\
\hline$>15 \mathrm{ml}$ & 30 & $42.9 \%$ \\
\hline \multicolumn{3}{|c|}{$\begin{array}{l}\text { Duration of organophosphate poisoning (Mean= } \\
\qquad 75.85 \pm 41.00 \text { minutes) }\end{array}$} \\
\hline$<60$ minutes & 28 & $40 \%$ \\
\hline$>60$ minutes & 42 & $60 \%$ \\
\hline \multicolumn{3}{|c|}{ Route of organophosphate poisoning } \\
\hline Oral & 52 & $74.3 \%$ \\
\hline Inhalation & 9 & $12.9 \%$ \\
\hline Skin & 9 & $12.9 \%$ \\
\hline
\end{tabular}

We calculated GCS of every registered patient in the emergency and divided the patients into two with one having a GCS of $<5$ and the other $>5$ with $81.40 \%$ having the latter as shown in Figure-1. The mean GCS came out to be 6.64 \pm 1.43 .

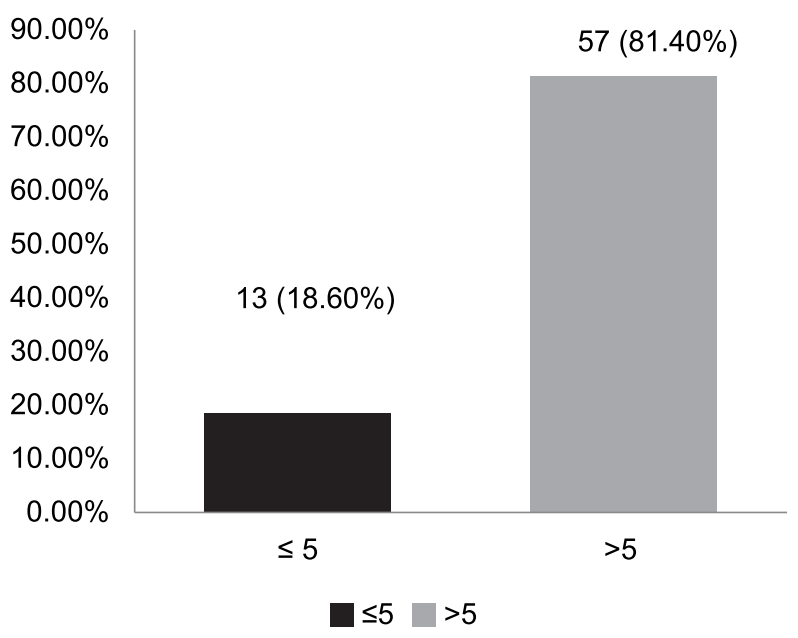

Figure-1. Glasgow coma score in patients of organophosphate poisoning.

The outcome of patients was presented in terms of mortality and it came out to be $17.10 \%$ as shown in Figure-2.

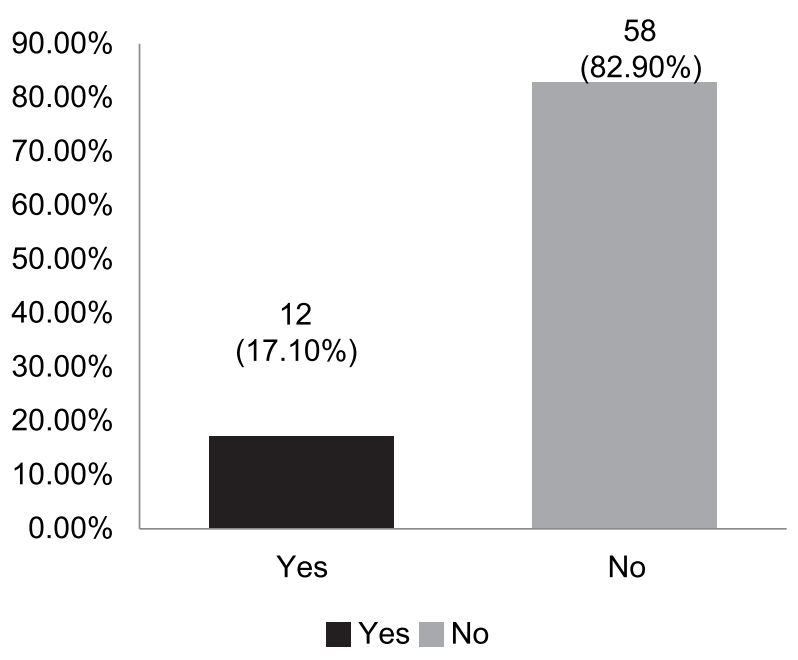

Figure-2. Mortality in patients of organophosphate poisoning.

We stratified the data by the differentcharacteristics of patients and determined the mortality in each group as shown in Table-II, but no significant association came out of it.

\begin{tabular}{|c|c|c|c|c|}
\hline & \multicolumn{2}{|c|}{ Mortality } & \multirow{2}{*}{ Total } & \multirow{2}{*}{ P-Value } \\
\hline & Yes $(n=12)$ & No $(n=58)$ & & \\
\hline \multicolumn{4}{|c|}{ Age groups } & \multirow{3}{*}{0.095} \\
\hline$\leq 30$ & $5(11.4 \%)$ & 39 (88.6\%) & 44 & \\
\hline$>30$ & 7 (26.9\%) & $19(73.1 \%)$ & 26 & \\
\hline \multicolumn{4}{|l|}{ Gender } & \multirow{3}{*}{0.764} \\
\hline Male & $8(18.2 \%)$ & 36 (81.8\%) & 44 & \\
\hline Female & 4 (15.4\%) & $22(84.6 \%)$ & 26 & \\
\hline \multicolumn{4}{|c|}{ Quantity of organophosphate poisoning } & \multirow{3}{*}{0.464} \\
\hline$\leq 15 \mathrm{~mL}$ & $8(20 \%)$ & 32 (80\%) & 40 & \\
\hline$>15 \mathrm{~mL}$ & 4 (13.3\%) & $26(86.7 \%)$ & 30 & \\
\hline \multicolumn{4}{|c|}{ Duration of organophosphate poisoning } & \multirow{3}{*}{0.437} \\
\hline$\leq 60 \min$ & $6(21.4 \%)$ & $22(78.6 \%)$ & 28 & \\
\hline$>60 \mathrm{~min}$ & $6(14.3 \%)$ & 36 (85.7\%) & 42 & \\
\hline \multicolumn{4}{|c|}{ Glasgow coma score } & \multirow{3}{*}{0.529} \\
\hline$\leq 5$ & $3(23.1 \%)$ & $10(76.9 \%)$ & 13 & \\
\hline$>5$ & 9 (15.8\%) & 48 (84.2\%) & 57 & \\
\hline \multicolumn{4}{|c|}{ Route of organophosphate poisoning } & \multirow{4}{*}{0.082} \\
\hline Oral & $12(23.1 \%)$ & $40(76.9 \%)$ & 52 & \\
\hline Inhalation & $0(0 \%)$ & $9(100 \%)$ & 9 & \\
\hline Skin & $0(0 \%)$ & $9(100 \%)$ & 9 & \\
\hline
\end{tabular}

Table-II. Association of mortality with other factors.

\section{DISCUSSION}

In this study, the use of GCS of the patient admitted 
in the emergency department as cases of OPP has been assessed as a predictor of mortality. Using a cutoff value of GCS as 5, the patients were divided into two groups, one with a highrisk having GCS $<5$ and another with a low-risk having GCS >5. The accuracy of the GCS score differed in terms of the quantity of poison taken and its duration. Mortality was seen in $17.10 \%$ of the patients. A study completed in 2011 found that the poisoning patients having below normal (<15) GCS score; had 44-90 times more chances to have complications and death as compared to the poisoning patients having normal GCS scores. $^{12}$ This GCS is also an independent predictor of survival in the general critically ill population and widely used prognostic intensive care scoring systems. ${ }^{13}$

In another study by Cander B et al., where different scoring systems were compared, GCS was found superior over other scoring systems in being easy to perform and not requiring complex physiologic parameters and laboratory methods. They also found that the death rate was $21.9 \%$ and only GCS correctly predicted a similar death rate among OPP patients. ${ }^{14}$ In this regard, the serial assessment of pseudo cholinesterase levels is an option but it is extremely expensive. And unavailability in many centers. ${ }^{15}$ Therefore, the GCS has been an effective clinical parameter helping to predict the outcome of OPP cases in the initial assessment.

A GCS score of $<13$ is believed to be the most sensitive predictor of serious complications (sensitivity $=79 \%$, specificity $=79 \%$ ) and is relatively good in predicting mortality following OP poisoning. ${ }^{16}$ Regarding outcome predictability of GCS among OPP patients; it was found by Sam KG et al. that among patients $(n=23)$ that presented with GCS <8; death occurred in four (17.39\%) patients. The mean GCS score of dying patients was $6.6 \pm 3.6$ while in the survivors the mean GCS score was $10.78 \pm 3.6$. They also found a significant negative correlation $(r=0.339)$ between the GCS scores and the incidence of mortality $(p<0.004) .{ }^{11}$ Furthermore, by Despande A et al. it was seen that the GCS score of 8.5 or lower was predictive of mortality, with $86 \%$ sensitivity and $72 \%$ specificity $(p<0.001)$. Identification of high death risk in OPP patients soon after presentation allows for more intensive monitoring and treatment. ${ }^{17}$ Male participants were predominant in our study, as it can be assumed with the kind of poisoning we have as a subject and this is similar to what Lin TJ et al. reported. ${ }^{18}$ On the other hand, we found that the majority of the cases of poisoning were through oral intake, this mirrors the work of other authors. ${ }^{18,19}$

The present study has a limitation in terms of usage of pulse oximetry or respiratory rate, as these parameters can be useful tools for predicting the outcomes of OP poisoning. Secondly, we only considered one of the outcome and it can be done in a better way in future studies. In an Indian study published in 2008, it was reported that the Peradeniya organophosphorus poisoning scale and level of serum Cholinesterase at the time presentation may prove helpful in the assessment of severity and hospital stay in organophosphate poisoning. ${ }^{20}$ While Sam KG et al. concluded that GCS, APACHE II ("Acute Physiology, Age, Chronic Health Evaluation II"), and (Predicted mortality rate) can be modeled in OPP. ${ }^{11}$ Though all of these ways are extensive and difficult to conduct in all patients, these have been done in the past, especially in the developing countries. In the light of abovementioned studies, it is recommended that further work should be done in our setup using bigger and more detailed assessment methods to predict the outcomes of OPP. Due to the short sample size, we did not get any statistically significant results, that can be made better by gathering more data from tertiary care hospitals from different regions of the country.

\section{CONCLUSION}

There are various methods for initial assessment of the mental and physical status of patients presenting with poisoning. This study concludes that the GCS can be a helpful and practical tool in assessing the mortality risk among the patients of OPP. However, because of the limited literature on the subject further studies are recommended to improve its validity.

Copyright 03 Feb, 2020. 


\section{REFERENCES}

1. Singh SP, Aggarwal AD, Oberoi SS, et al. Study of poisoning trends in north India--a perspective in relation to world statistics. J Forensic Leg Med. 2013; 20(1):14-8.

2. Lee BK, Jeung KW, Lee HY,et al. Mortality rate and pattern following carbamate methomyl poisoning. Comparison with organophosphate poisoning of comparable toxicity. Clin Toxicol (Phila). 2011; 49(9):828-33.

3. Naik KR, Saroja AO, Hesarur N, et al. Prospective hospitalbased clinical and electrophysiological evaluation of acute organophosphate poisoning. Ann Indian Acad Neurol. 2019; 22(1):91-5.

4. WHO recommended classification of pesticides by hazard and guidelines to classification 2009. World Health Organization. Geneva; 2009.

5. Shaikh MA, Ujjan ID, Memon SH. Evaluation of patients with organphosphorus poisoning at a tertiary care hospital of Sindh. Med Channel. 2011; 17:51-3.

6. Balouch G, Yousfani A, Jaffery $M$, et al. Electrocardiographical manifestations of acute organophosphate poisoning. World Appl Sci J. 2012; 16(8):1118-22.

7. Teasdale G. Forty years on: Updating the Glasgow Coma Scale. Nursing Times; 110: 42, 12-16. Nurs Times. 2014; 110(42):12-6.

8. Seeley H, Maimaris C, Allanson J,et al. Service use following attendance at an emergency department with an head injury: A 6-month survey. Emerg Med J. 2014; $31(9): 724-9$.

9. Borron SW, Burns MJ, Haddad LM, et al. Clinical management of poisoning and drug overdose. 4th ed. Philadelphia: Saunders/Elsevier; 2007. 1559 p.

10. Shadnia S, Okazi A, Akhlaghi N, et al. Prognostic value of long QT interval in acute and severe organophosphate poisoning. J Med Toxicol. 2009; 5(4):196-9.
11. Sam KG, Kondabolu K, Pati D, et al. Poisoning severity score, APACHE II and GCS: Effective clinical indices for estimating severity and predicting outcome of acute organ phosphorus and carbamate poisoning. $J$ Forensic Leg Med. 2009; 16(5):239-47.

12. Eizadi Mood N, Sabzghabaee AM, Yadegarfar G,et al. Glasgow coma scale and its components on admission: Are they valuable prognostic tools in acute mixed drug poisoning? Crit Care Res Pract. 2011; 2011:952956.

13. Rapsang AG, Shyam DC. Scoring systems in the intensive care unit: A compendium. Indian J Crit Care Med. 2014; 18(4):220-8.

14. Cander B, Dur A, Yildiz M, et al. The prognostic value of the Glasgow coma scale, serum acetylcholinesterase and leukocyte levels in acute organophosphorus poisoning. Ann Saudi Med. 2011; 31(2):163-6.

15. Crane AL, Abdel Rasoul G, Ismail AA, et al. Longitudinal assessment of chlorpyrifos exposure and effect biomarkers in adolescent Egyptian agricultural workers. J Expo Sci Environ Epidemiol. 2013; 23(4):35662.

16. Davies JOJ, Eddleston M, Buckley NA. Predicting outcome in acute organophosphorus poisoning with a poison severity score or the Glasgow coma scale. QJM. 2008; 101(5):371-9.

17. Deshpande A, Gaikwad N, Deshpande S. Study of Glasgow Coma Scale Score and QTc interval in prognosis of organophosphate compound poisoning. Indian J Clin Med. 2012; 3:25-31.

18. Lin TJ, Walter FG, Hung DZ, et al. Epidemiology of organophosphate pesticide poisoning in Taiwan. Clin Toxicol (Phila). 2008; 46(9):794-801.

19. Adinew GM, Asrie AB, Birru EM. Pattern of acute organophosphorus poisoning at University of Gondar Teaching Hospital, Northwest Ethiopia. BMC Res Notes. 2017; 10(1):149.

20. Rehiman S, Lohani SP, Bhattarai MC. Correlation of serum cholinesterase level, clinical score at presentation and severity of organophosphorous poisoning. JNMA J Nepal Med Assoc. 2008; 47(170):47-52. 


\begin{tabular}{|c|l|l|l|}
\hline \multicolumn{2}{|c|}{ AUTHORSHIP AND CONTRIBUTION DECLARATION } \\
\hline Sr. \# & \multicolumn{1}{|c|}{ Author(s) Full Name } & \multicolumn{1}{|c|}{ Contribution to the paper } & Author(s) Signature \\
\hline 1 & Muhammad Ali & Manuscript writing. \\
\hline 2 & Vinod Kumar & Manuscript preparation. \\
\hline 3 & Shafique Ahmed & Data analysis. \\
\hline 5 & Hina Iram & Data collection. \\
\hline 6 & Rukhsana Abdul Sattar & Manuscript review.
\end{tabular}

